\title{
Tumor glómico de la mano: una localización extradigital poco habitual
}

\author{
Francisco Albaladejo', P. Sánchez Angulo', M. Hernández Torralba', F. Martínez \\ Díaz ${ }^{2}$ M. V. Redondo Carazo . \\ 'Unidad de Cirugía de la Mano y Microcirugía. Servicio de Cirugía Ortopédica y Traumatología. ²Servicio de Anatomía \\ Patológica. ${ }^{3}$ Servicio de Radiodiagnóstico. Hospital General Universitario Reina Sofía. Murcia. España.
}

\begin{abstract}
RESUMEN
Los tumores glómicos representan el $2 \%$ de los tumores de la mano. Con mayor frecuencia se trata de tumoraciones únicas, situadas a nivel subungueal, a veces periungueal o en el tejido subcutáneo del pulpejo de los dedos. Es infrecuente pero pueden afectarse varios dedos en el mismo paciente.

La localización extra digital es poco habitual, haciendo aún más difícil su diagnóstico.

Se presenta el caso de una paciente de 45 años con una tumoración redondeada de consistencia dura, situada en la base del pulgar a nivel de la eminencia tenar, de 5 años de evolución, que presentaba dolor a la presión e intolerancia al frío. Su extirpación solucionó la clínica y el estudio anatomo-patológico confirmó el diagnóstico.
\end{abstract}

Palabras clave: Tumor. Mano. Tumor glómico. Glomangioma.

\begin{abstract}
We present the case of a 45 year old female with a five year history of pain at the base of her thumb, in the thenar eminence, where she has a firm and spherical mass. The patient complained of sharp pain whenever pressure The glomus tumor accounts for $2 \%$ of tumors in the hand. They are usually isolated lesions and typically located in the subungueal region. Sometimes, they are located periungueal or subcutaneous, beneath the pulp of the digits. Infrequently, they involve more than on digit in the same patient. The extradigital location is rare, making the diagnosis even more difficult.

The lump was applied to the base of her thumb with cold sensitivity.

The surgical excision was curative and the histopathology was consistent with the pre-operative diagnosis of glomus tumor.
\end{abstract}

Key words: Tumor. Hand. Glomus tumor. Glomangioma.

El tumor glómico o glomamgioma, representa el 2\% de los tumores de la mano.

Descrito por Wood en 1812 denominándolo "tubérculo subcutáneo doloroso", fue Mason' en 1924, quien lo describe con mayor precisión, llamándolo "tumor del glomus neuromioarterial"'.

En los vasos glómicos se identifican las características células epiteliodes del tumor glómico, derivadas de los pericitos de Zimmerman.

Se trata frecuentemente de tumoraciones únicas, que se sitúan a nivel subungueal, a veces periungueal o en el tejido subcutáneo del pulpejo de los dedos. Están descritos los tumores glómicos múltiples en varios dedos del mismo paciente.

Son tumores difíciles de diagnosticar, siendo pacientes que suelen visitar la consulta de distintos especialistas hasta conseguir su diagnóstico. En la literatura aparece reflejada una media de siete años desde el inicio de los síntomas hasta su diagnóstico, habiéndose descrito un caso de 40 años de evolución ${ }^{2}$. En la serie de Van Geertruyden y colos ${ }^{3}$, sobre cincuenta y un casos, la duración de los síntomas antes de recibir el tratamiento, fue de diez años.

Clínicamente se manifiestan con la asociación de tres signos clínicos, que para Carroll y Berman ${ }^{4}$ son patognomónicos:

I. Dolor espontáneo, muy localizado, pudiendo irradiar al antebrazo y hombro, con bruscos paroxismos hiperálgicos.

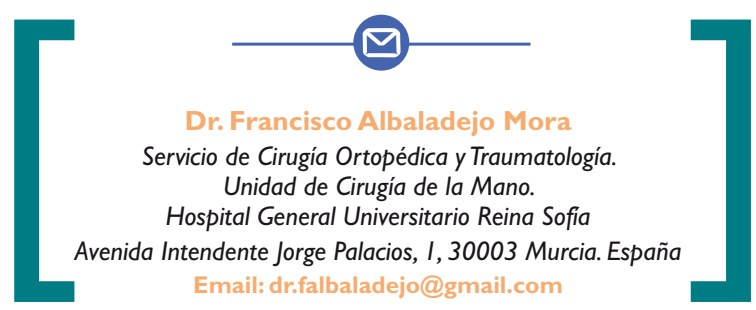


2. Dolor intenso provocado al tocarlo, incluso suavemente.

3. Intolerancia a los cambios de temperatura y en particular al frío.

A veces el tumor es visible como una mancha violácea, sub o periungueal que nos orienta hacia el diagnóstico.

Histológicamente se clasifican en tipo I o mucoidehialino, tipo II o sólido y tipo III o glomangioma.

Se ha utilizado para su diagnóstico la radiología simple, donde puede apreciarse en los casos de larga evolución una muesca o impronta sobre la falange distal, la ultrasonografía, la transiluminación y la arteriografía también pueden ayudar al diagnóstico, pero actualmente el método de elección es la resonancia magnética $R M^{5}$ que delimita la localización y el tamaño del tumor, resultando de gran utilidad en localizaciones múltiples o poco frecuentes.

El tratamiento consiste en la extirpación completa con lo que se obtiene la curación, con desaparición del dolor. La recidiva es posible por exéresis incompleta, siendo excepcional la malignización ${ }^{6,7}$.

Se presenta el caso clínico de un tumor glómico atípico por su localización extra digital que es excepcional.

\section{CASO CLÍNICO:}

Se presenta el caso de una mujer de 44 años, licenciada y educadora de profesión, con antecedente de cirugía de pulgar en resorte de la mano izquierda hace 10 años. Visita al Médico de Familia por presentar dolor en cara palmar de la mano izquierda que aumenta con el frío.

Acude a consulta externa, tras ser vista por Dermatología y Cirugía General, presentando una tumoración pequeña y dura en cara palmar de la mano, proximal al pliegue de flexión metacarpofalangico del pulgar, de 5 años de evolución, siendo visible una coloración rojiza que coincide con la tumoración (2) Figura I. Relata intolerancia al frío y dolor cuando se le presiona. Se realiza estudio ecográfico que informa de tumoración de $8 \mathrm{~mm}$, que contiene un vaso polar (2) Figura 2.

Ante la sospecha clínica de tumor glómico, aunque no es una localización habitual, se interviene quirúrgica-

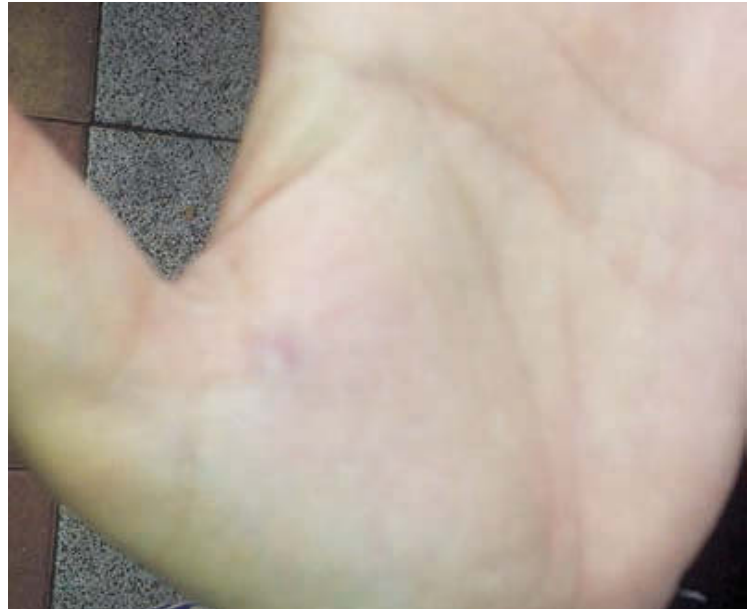

Figura I. Tumoración de color violáceo en la base del pulgar de la mano izquierda.

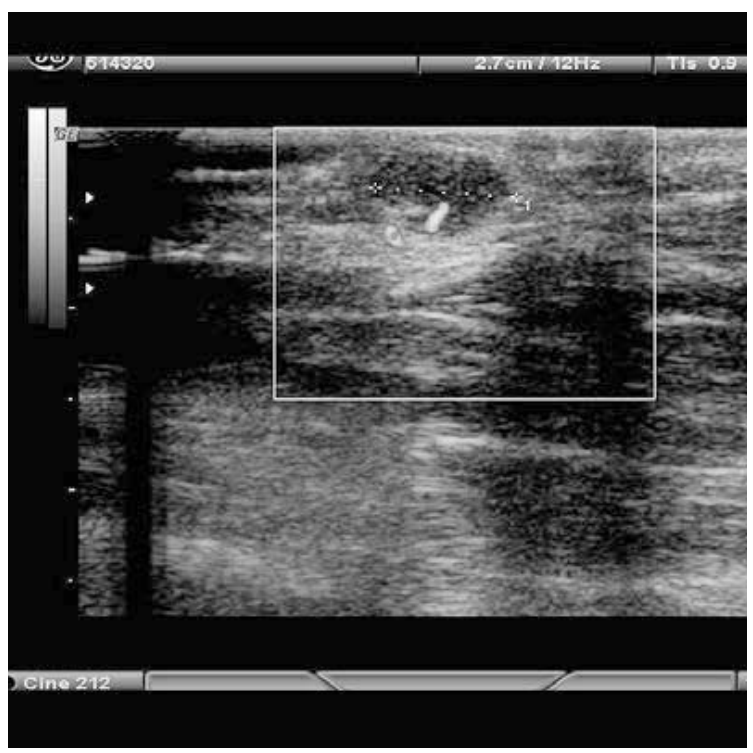

Figura 2. Imagen de ecografía que muestra una lesión sólida hipoecoica, nodular de $8 \mathrm{~mm}$, con un vaso polar.

mente, encontrando una tumoración dura, nacarada (siempre es rojiza, pero por la isquemia y el revestimiento capsular, aparece nacarada), redondeada de I cm de tamaño (a) Figuras 3 y 4, que se envía para estudio Anatomo-Patológico A.P.

El estudio (2) Figuras 5 y 6 presenta la isguiente descripción macroscópica: nódulo de tejido de 0,8 mm de coloración grisácea, bien delimitado y consistencia elástica blanda. Descripción microscópica: la tumoración está constituida por una proliferación de células de pequeño tamaño, núcleos redondeados u ovoideos, sin nucléolo y sin figuras de mitosis que se dis- 


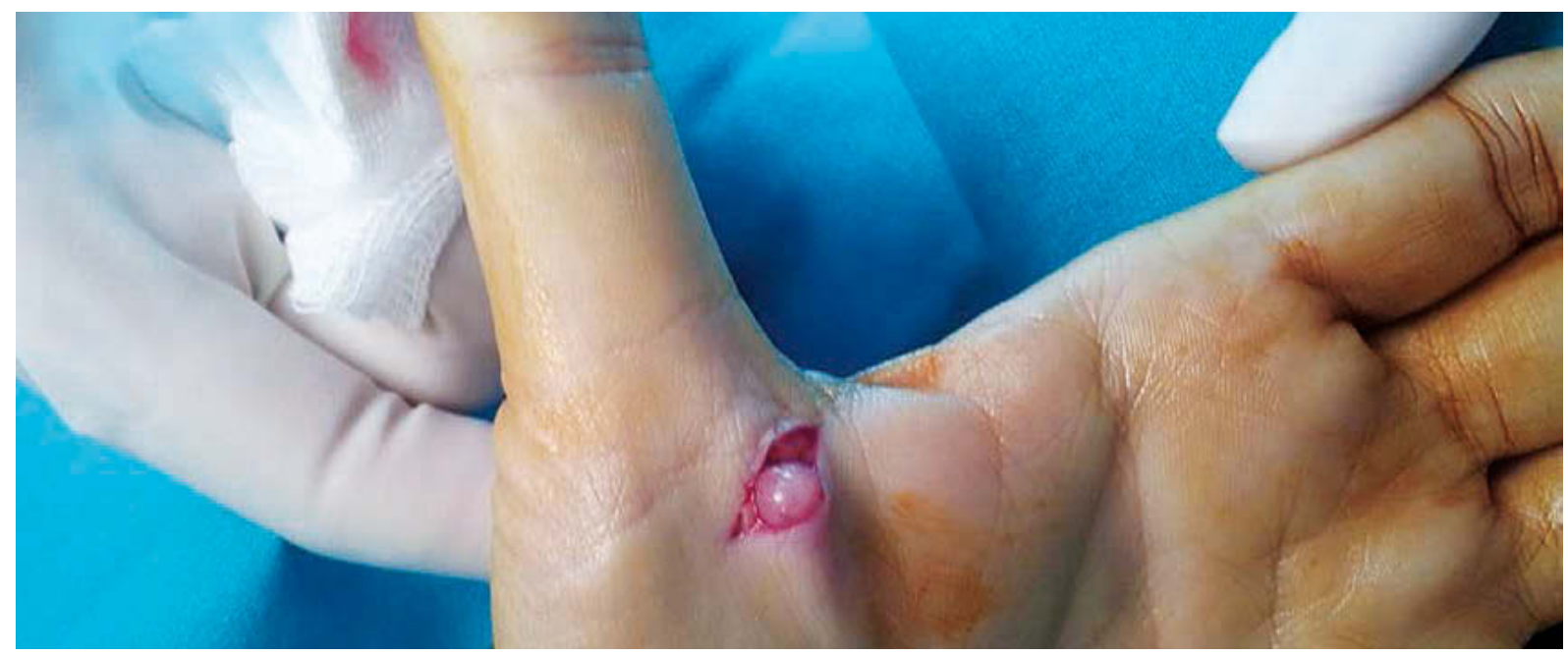

Figura 3. Imagen intraoperatoria que muestra la tumoración, redondeada y color nacarado, situada a nivel subcutáneo en la base del pulgar.
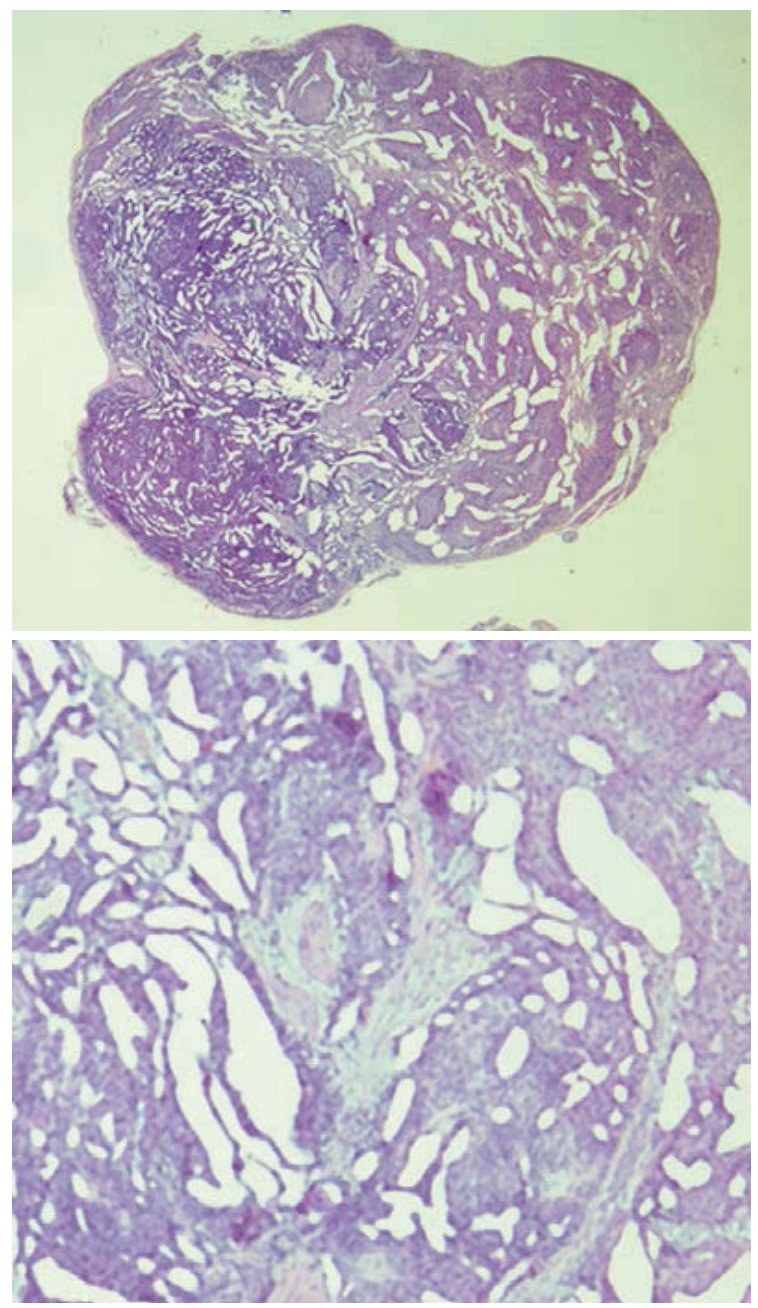

Figuras 5 y 6. Estudio histológico de la tumoración con tinción de Hematoxilina-eoxina.

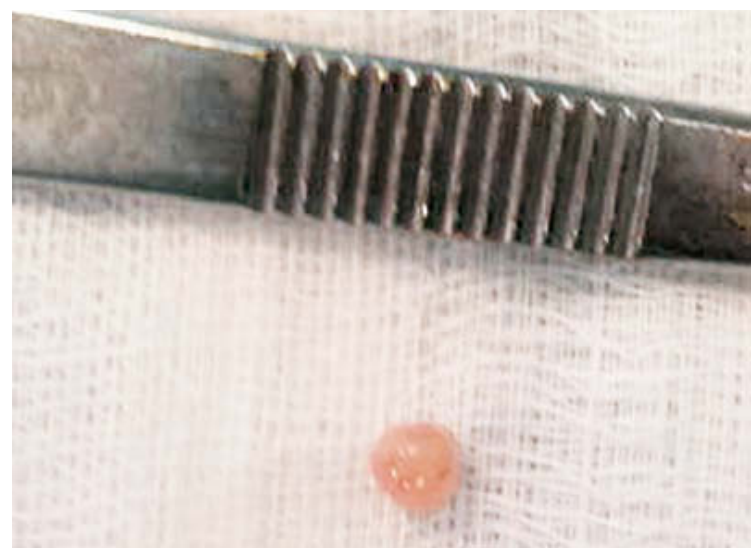

Figura 4. Tumoración redonda de $1 \mathrm{~cm}$. Imagen macroscópica tras la extirpación.

ponen alrededor de hendiduras vasculares. Por fuera se dispone una fina cápsula fibrosa, confirma el diagnóstico de tumor glómico.

Actualmente la paciente se encuentra asintomática desde la exéresis, habiendo transcurrido 20 meses desde la intervención.

\section{DISCUSIÓN}

El tumor glómico es de difícil diagnóstico cuando se localiza a nivel digital sub o periungueal ${ }^{1-3}$, pero más aún cuando la localización, como en nuestro, caso es atípica, haciendo difícil la sospecha clínica. 
La localización extra digital es poco frecuente. Existen casos aislados como el descrito por Takei ${ }^{8}$, en el tercio distal del antebrazo, o el descrito por Irisarri y Capomassi', sobre el dorso de la cabeza del quinto metacarpiano. Existen otros de localización intraósea en la falange distal de los dedos largos, 10. También se han publicado en el nervio radial, y en el nervio colateral del pulgar.

Su diagnóstico es difícil, haciendo que los pacientes consulten a diferentes especialistas, incluso se ha pensado en casos que se trata de un trastorno psicológico, siendo enviado el paciente al psiquiatra. Van Geertruyden y cols. ${ }^{3}$ publica una serie de 5 I casos con una duración media de los síntomas antes de recibir tratamiento de 10 años.

Para su diagnóstico se ha utilizado la ultrasonografía, la transiluminación, la termografía dinámica, y la ecografía pero actualmente han sido desplazados por la RM ${ }^{5}$, que proporciona imágenes mas nítidas en cuanto a tamaño y ayuda a la localización, siendo de mayor utilidad en localizaciones atípicas y múltiples.

Esta descrita la malignización, Gould y cols." observó 6 casos en su serie, localizándose uno en la mano. Wetherington y cols. ${ }^{7}$ refiere un glomangio-sarcoma en el pulgar con extirpación amplia y sin recidiva al año de su extirpación. Pérez de la Fuente y cols. ${ }^{6}$ refiere otro caso localizado en la eminencia hipotenar, sin recidiva tras 18 meses de su extirpación.

Todos los autores coinciden en que el tratamiento es curativo en todos los casos, siempre que se consiga la exéresis completa de la tumoración, con la consecuente desaparición del dolor. Es posible la recidiva, pero suele deberse a la extirpación incompleta del tumor. Blasco y cols.' ${ }^{2}$ describe una recidiva de una tumoración glómica en la falange distal del $4^{\circ}$ dedo, 30 años después de su extirpación inicial.

Aunque vemos que el mayor número de publicaciones hace referencia a un caso concreto, existen series con un alto número de pacientes ${ }^{3,4}$. Estas series tan numerosas nos llevan a la reflexión de que no es una tumoración tan rara, sino que lo difícil sea posiblemente su diagnóstico. Debemos pensar en el tumor glómico ante la presencia de la triada característica de hipersensibilidad al frío, al tacto y dolor paroxístico.

\section{CONFLICTOS DE INTERESES}

Los autores declaran no tener conflictos de intereses

\section{BIBLIOGRAFIA}

I. Irisarri C, Capomassi M. Tumores de la mano. Madrid: Editorial Médica Panamericana; 2009.

2. Leclerq C, Glicestein J.Tumeurs des parties molles de la main. En:Tubiana R, (Ed). Traite de Chirurgie de la Main. Paris Masson; 1995 p. 768-9.

3. Van Geertruyden J, Lorea P, Goldschmidt D, de Fontaine S, Schuind F, Kinnen L, et al. Glomus tumours of the hand. A retrospective study of 51 cases. J Hand Surg Br. 1996;2 I (2):257-60.

4. Carroll RE, Berman AT. Glomus tumors of the hand: review of the literature and report on twentyeight cases. J Bone Joint Surg Am. 1972;54(4):691 703.

5. Al-Qattan MM, Al-Namla A, Al-Thunayan A, AlSubhi F, El-Shayeb AF. Magnetic resonance imaging in the diagnosis of glomus tumours of the hand. J Hand Surg Br. 2005;30(5):535-40.

6. Perez de la Fuente T,Vega C, Gutiérrez Palacios A, Sánchez Lorenzo J, González Sarasua J. Glomangiosarcoma of the hypothenar eminence: a case report. Chir Main. 2005;24 (3-4): 199-202.

7. Wetherington RW, Lyle WG, Sangueza OP. Malignant glomus tumor of the thumb: a case report. J. Hand Surg Am. 1997;22 (6): 1098- 102.

8. TakeiTR, Nalebuff EA. Extradigital glomus tumour.J. Hand Surg Br. 1995;20(3):409-12.

9. Proubasta I, del Pozo Manrique P.Tumor glómico digital.Importancia de la RNM en su diagnóstico. Avances Traum. 1997;27(1):29-31.

10. Rus A, Gastaldi E. Tumor glomico intraoseo (Révision a propósito de un caso). Rev Esp Cir Ost. 1988:233-7.

II. Gould EW, Manivel JC, Albores-Saavedra J, Monforte H. Locally infiltrative glomus tumors and glomangiosarcomas. A clinical, ultrastructural, and immunohistochemical study. Cancer. 1990;65(2):310-8.

12. Blasco C, Arguelles S, Silvestre A, Gomar F. Tumor glómico intraóseo Caso clínico. Rev Esp Cir Ost. 1999;34(197):6 |-4. 